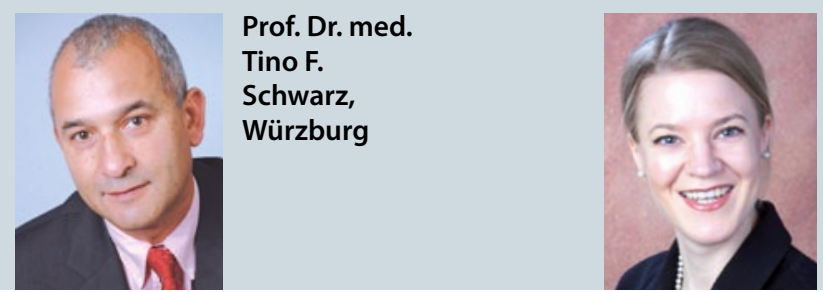

PD Dr. med.

Petra Stute,

Bern

bis zur Menopause ließ sich mithilfe der Cox-Regressionsanalyse untersuchen.

48 Frauen erreichten innerhalb des Beobachtungszeitraums die Postmenopause. Alter, AMH-Wert und AFC korrelierten dabei signifikant $(\mathrm{p}<0,001)$ mit der Zeitdauer bis zur Menopause und erlaubten häufig das Menopausenalter exakt vorherzusagen. Nach Adjustierung für den Faktor „Alter“ galt dies nur noch für $\mathrm{AMH}$.

Broer SL et al., Anti-mullerian hormone predicts menopause: a long-term follow-up study in normoovulatory women. J Clin Endocrinol Metab 2011; 96: 2532-9

Kommentar: Da in der vorliegenden Studie die Beziehung zwischen AMH und dem Zeitpunkt der zukünftigen Menopause nur bei normoovulatorischen Frauen untersucht wurde, haben die Ergebnisse nur bei dieser Patientinnengruppe Aussagekraft. Für die jüngere ovulatorische Frau, die ihr Menopausenalter abschätzen möchte, hat die Methode demnach einen guten prädiktiven Wert. Die prädiktiven Bereiche des Menopausenalter umfassen dabei für alle AMH-Perzentile eine Zeitspanne von zehn Jahren. Dagegen besitzt AMH keinen prädiktiven Wert für Frauen mit unregelmäBigen Zyklen in einem Alter von fast 50 Jahren. Gerade diese Frauen würden von der exakten Vorhersage des Menopausenalters profitieren. Eine weitere Limitation der Studie: In den verschiedenen Kohorten wurden unterschiedliche Verfahren zur AMH-Bestimmung eingesetzt. Dennoch: Die Studie ist ein Schritt in die richtige Richtung. Und sie untermauert erneut die Bedeutung von AMH als bestem Marker für die Eizellreserve. $\quad P D D$ r. Petra Stute

\title{
Türöffner für Spermien bahnt Candida den Weg
}

\author{
Um die Zeit des Eisprungs herum sind Frauen besonders anfällig für \\ Infektionen. Schuld daran sind, zumindest indirekt, die üblichen Verdächtigen: \\ Männer - respektive deren Spermien.
}

$\mathrm{K}^{\mathrm{o}}$ men normalerweise nicht gern gesehen, die Aufräumarbeit erledigt die Immunabwehr. Andererseits steht dies der Fortpflanzung arg im Weg, denn hier ist die Anwesenheit fremder Zellen geradezu obligat. Aus diesem Grund ist die Natur auf einen Trick verfallen: Während und nach der Ovulation wird die körpereigene Immunpolizei an die Kette gelegt; Spermien wird so das Überleben und der Menschheit der Fortbestand gesichert. Doch der Trick hat einen Haken. Denn der Schutz für die Spermien rettet auch manchem Krankheitserreger das Leben - die Gefahr von Infektionen durch Candida, Chlamydien, Toxoplasmen und Listerien steigt. Ein spanisch-österreichisches Forscherteam hat nun womöglich aufgedeckt, woran das liegt.

Die Wissenschaftler untersuchten die Hypothese, wonach das periovulatorisch erhöhte Infektionsrisiko mit dem in diesem Zeitraum maximal erhöhten Estradiolspiegel zusammenhängt. Zu diesem Zweck behandelten sie ovariektomierte Mäuse mit dem Hormon und setzten sie danach Candida albicans aus. Estradiolbehandelte Tiere erwiesen sich als anfälliger für eine Pilzinfektion als unbehandelte Mäuse. Ihre Th17-Immunantwort fiel schwächer aus. Die Forscher entdeckten, dass das Estradiol auf die dendritischen Zellen zielt. Derart behindert, konnten sie nur noch eingeschränkt Interleukin-23 produzieren und die Th17-
Anwort weniger effizient triggern - Candida albicans hatte leichtes Spiel.

„Wenn Sie das nächste Mal eine Frau sagen hören, Männer machten sie krank, können Sie diesen Befund der Liste ihrer Gründe anfügen“, lautet der trockene Kommentar von John Wherry, dem stellvertretenden Chefredakteur der Zeitschrift "Journal of Leukocyte Biology“, zur Estradiolstudie. „Was den Spermien erlaubt, lange genug zu leben, um die Eizelle zu befruchten, öffnet auch anderen Arten von Infektionen die Tür." Bestätigt wird damit zugleich ein alter Verdacht: Das Leben ist eine ansteckende, sexuell übertragene Krankheit, die noch dazu meist tödlich endet.

Dr. Robert Bublak

Relloso M et al. Estradiol impairs the Th17 immune response against Candida albicans. J Leukoc Biol 2012; 91: 159-65

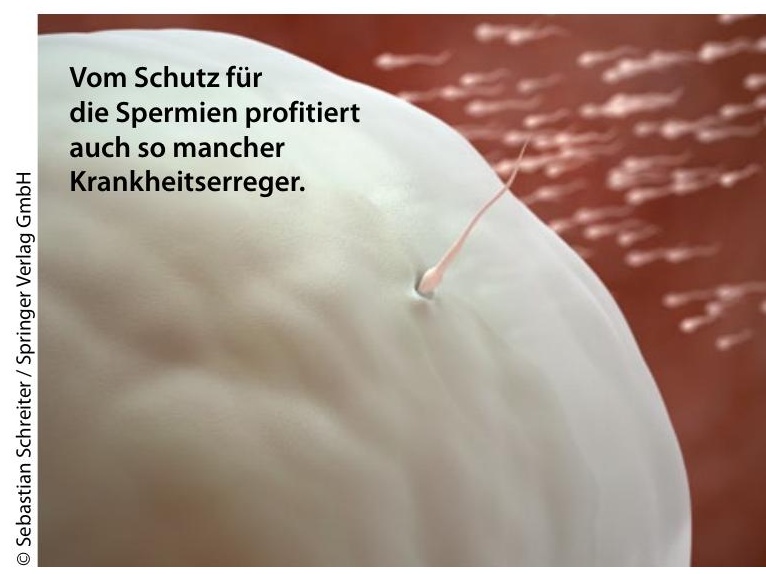

